\title{
Surgical Closure of Nasoseptal Defects: Postoperative Patient Satisfaction
}

\author{
Florian Bast $^{\mathrm{a}}$ Anna Heimer ${ }^{\mathrm{b}}$ Thomas Schrom ${ }^{\mathrm{b}}$ \\ ${ }^{a}$ Campus Virchow-Klinikum, Department of Otorhinolaryngology, University Hospital Charité Berlin, Berlin, and \\ ${ }^{\mathrm{b}}$ Clinic for Otorhinolaryngology, Head and Neck Surgery/Plastic Surgery, Helios Clinics Bad Saarow, \\ Bad Saarow, Germany
}

\section{Key Words}

Nasoseptal defect $\cdot$ Bridge flap technique $\cdot$ Quality of life $\cdot$

Glasgow benefit inventory $\cdot$ SNOT-20 GAV

\begin{abstract}
Background: Nasoseptal defects are rather rare. The current therapy of choice is surgical closure. Objective clinical factors prove the efficacy of surgical intervention; however, to establish the quality of the procedure patients' subjective quality of life assessments must also be considered. Methods: 31 patients participated in the study. The operation was always carried out according to the superior and inferior bipedicle bridge flap technique according to Schultz-Coulon. The patient survey was performed retrospectively, and patient satisfaction was evaluated using two different questionnaires: the Sino-Nasal Outcome Test 20 German Adapted Version (SNOT-20 GAV SDT) expanded by Neumann [2010], and the Glasgow Benefit Inventory (GBI). Results: Evaluation of the $\mathrm{GBI}$ revealed significant improvements in the total score and the subscore 'general health' and therefore an improved quality of life. Evaluation of the SNOT-20 GAV SDT showed a significant reduction in the total score and a significant reduction in the primary nasal symptoms as well as in the items typical of septum defects and therefore less dis-
\end{abstract}

comfort caused by the nasoseptal defect. Conclusion: As the gold standard for treatment of septum perforation, successful surgical closure leads to an improvement in subjective quality of life.

Copyright $\odot 2012$ S. Karger AG, Basel

\section{Introduction}

A nasoseptal defect is a relatively rare condition, with a prevalence of $0.9 \%[1,2]$. In terms of Germany's current population of approximately 81.7 million, this means more than 73,000 people have some degree of discomfort due to a three-layered nasoseptal defect. About $2 / 3$ of such defects are iatrogenic in origin. The high number of nasoseptal defects can be in part be deduced from the estimated number of septoplasties carried out each year in Germany. Stange et al. [2] estimate over 250,000 operations per year, with a postoperative perforation rate of $1.5-5 \%$ according to the literature. About $1 / 3$ of the perforations are spontaneous defects [1-7].

The study was presented at the German ENT Meeting, June 4, 2011, Freiburg, Germany.

\section{KARGER}

Fax +41613061234

E-Mail karger@karger.ch

www.karger.com
(C) 2012 S. Karger AG, Basel

0301-1569/12/0746-0299\$38.00/0

Accessible online at:

www.karger.com/orl
Dr. med. Florian Bast

University Hospital Charité Berlin

Department of Otorhinolaryngology, Campus Virchow-Klinikum

Augustenburger Platz 1, DE-13353 Berlin (Germany)

E-Mail Florian.Bast@ charite.de 
Thus, a nasoseptal defect is a condition regularly treated by otorhinolaryngologists. If there are no complaints, no treatment is necessary, and when the perforation causes only mild symptoms, a conservative treatment involving careful nasal hygiene can be sufficient. If more serious complaints occur such as whistling noise during inhalation, excessive endonasal incrustations with impaired nasal breathing, pain or recurring epistaxis then a perforation closure is desirable. This can be done surgically or via epithesis [7-13]. The size of the perforation allowing, we favor the three-layered surgical closure with the Schulz-Coulon bridge flap technique. More than 40 methods for closing a nasoseptal defect are described in the literature [2, 7], from enlarging the defect (according to Imperatori [14]) to minimizing symptoms, to the surgically very complex supraorbital flap or the forehead flap [15]. So far a high perforation closure rate (over 90\%) has only been shown in a large patient population for SchulzCoulon's bridge flap technique, which unites Seiffert's idea for the bridge flap and Seeley's principle of expanded bilateral mucous membrane mobilization $[2,10,11]$. Still, the surgical reconstruction of a nasoseptal defect is one of the most difficult rhinosurgical procedures. The success of a medical or surgical intervention can not only be judged according to the degree of technical success; changes in the patient's quality of life resulting from such an intervention must also be considered [16-19]. In this connection, various validated quality of life assessments are currently available. Some general assessment instruments are the 'Short Form 36 Health Survey', the 'Short Form 12 Health Survey', the 'European Quality of Life 5 Dimensions' as well as the 'Glasgow Benefit Inventory'. In addition, there are also special assessments for sinonasal complaints, among them the 'Nasal Symptom Questionnaire', the 'Rhino-Sinusitis Disability Index', the 'General Nasal Patient Inventory', the 'Rhinosinusitis Quality of Life Survey' and the 'Sino-Nasal Outcome Test 20' [1925].

The Glasgow Benefit Inventory (GBI) is especially designed for ENT health problems and procedures. The survey, composed of 18 questions, was introduced in 1996 as a postntervention assessment and reflects changes in health conditions after surgical or conservative treatments. Health status is defined as the general perception of one's own health, including all psychosocial, social and physical aspects. The GBI is a postintervention assessment designed for maximum sensibility to changes in health and has often appeared in the literature [21, 26, 27].

The SNOT-20 is a shortened form of the Rhinosinusitis Outcome Measure (RSOM-31) introduced by Piccirillo
[25], containing general and rhinosinusitis-related questions. The SNOT-20 queries 20 symptoms of rhinosinusitis, which are divided into 5 subgroups: nasal, paranasal, sleeping, social and emotional. In recent years, the SNOT20 has been increasingly used to assess quality of life in patients with chronic rhinosinusitis. In 2008, Baumann introduced a slightly altered German version of the SNOT20: The Sino-Nasal Outcome Test 20 German Adapted Version (SNOT-20 GAV). To the SNOT-20 GAV, the subscores primary nasal symptoms, secondary rhinogenic symptoms and general quality of life were added in order to facilitate a more specific evaluation. The SNOT-20 GAV is at the moment the only German instrument for measuring health-related quality of life in patients with chronic rhinosinusitis [28]. In order to evaluate quality of life in patients following closure of a nasoseptal defect via obturator, the 20 questions on the SNOT-20 GAV were expanded to include 5 nasoseptal defect-specific questions introduced by Neumann in 2010 regarding bleeding, whistling noise, pain, temperature perception and foreign body sensation (SNOT-20 GAV SDT) [12, 21, 25].

The goal of the current study is to validate quality of life following surgical septum perforation closure using the GBI and SNOT-20 GAV expanded to included questions concerning nasoseptal defect symptoms.

\section{Materials and Methods}

After approval by the Medical Association and Ethics Committee in Cottbus, Brandenburg, the study was financed by the HELIOS Research support ID 011398.

All patients who underwent a three-layer nasoseptal defect operation in our hospital also underwent a follow-up examination as outpatients. The study included all 46 patients operated between September 2008 and June 2010. The postoperative observation period was between 3 months and 2 years, in the mean 7 months. In this time just 1 patient showed a small and symptomfree post-operative residual defect. The patient survey was conducted retrospectively using two standardized questionnaire: the Sino-Nasal Outcome Test 20 German Adapted Version (SNOT-20 GAV SDT) expanded by Neumann in 2010 and the GBI. The questionnaires and a reminding letter 6 weeks later were sent via mail. The rate of return was over $67 \%$; 31 patients ( 20 men and 11 women, average age 49.9) were included in the study.

The operation was always performed by the same surgeon and using the superior and inferior bipedicle bridge flap technique according to Schultz-Coulon under general anesthesia. The silicone splints inserted at the end of the operation were removed 10 days after the operation in our outpatient clinic. Dexpanthenol nasal ointment and nose drops to reduce swelling were prescribed. $\mathrm{Pa}$ tients were instructed not to blow their nose for 6 weeks.

As part of the statistical analysis, the data was first checked for normal distribution with the help of the Shapiro-Wilk test in or- 


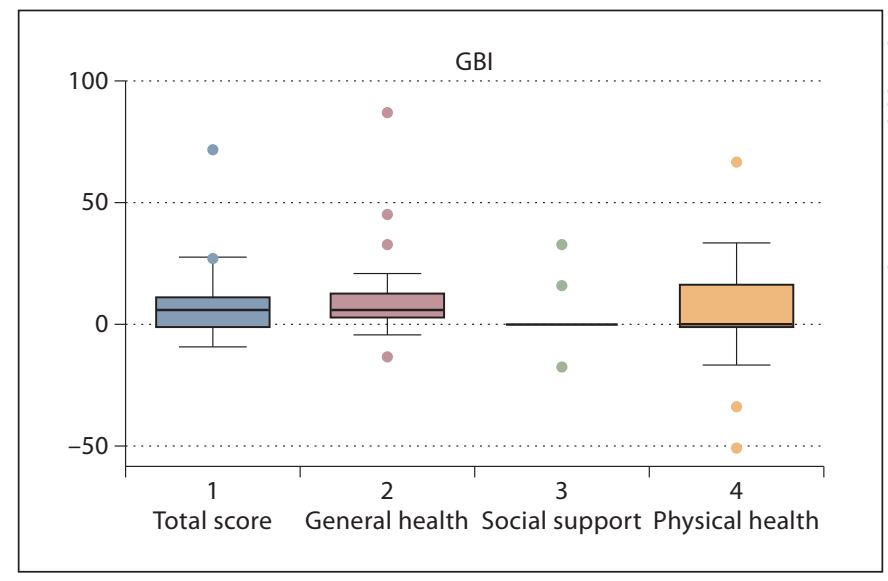

Fig. 1. Scores of the GBI with a significant improvement in the total score and in the subscore 'general health'.

der to compare the distribution to a metrical variable of two combined random samples. If the normal distribution assumption was rejected, the Wilcoxon rank-sum test was used, otherwise the $t$ test for combined random samples. The Rand homogeneity test was used to compare the distribution of categoric or ordinal variables of combined random samples. All tests (except ShapiroWilk; $\mathrm{p}=0.1$ ) were calculated bilaterally at $\mathrm{p}=0.05$.

\section{Results}

A significant improvement in the total score and in the subscore 'general health' resulted in the analysis of the 18 questions on the GBI. The subscore 'physical health' also improved postoperatively although no significant level was reached. The evaluation of the point 'social support' showed no difference (fig. 1). A score bigger 0 shows an improvement of the evaluated scores after closure of the nasoseptal defect and thus an improved quality of life. A score equal to 0 shows no change and a score less than 0 shows an impairment of the evaluated scores after closure of the nasoseptal defect.

The analysis of the SNOT-20 GAV SDT showed in total a significant reduction of the total score (GSD) with $\mathrm{p}=$ 0.018 as well as a significant reduction in both the primary nasal symptoms (PNS) category with $\mathrm{p}=0.001$ and the nasoseptal defect-related symptoms (SDT) with $\mathrm{p}=0.017$ and therefore reduced ailments coursed by the nasoseptal defect. In the subscores secondary rhinogenic symptoms (SRS) and general quality of life (ALQ) a reduction of scores in the middles range was evident postoperatively, although no significant level was reached (fig. 2).

Surgical Closure of Nasoseptal Defects:

Postoperative Patient Satisfaction

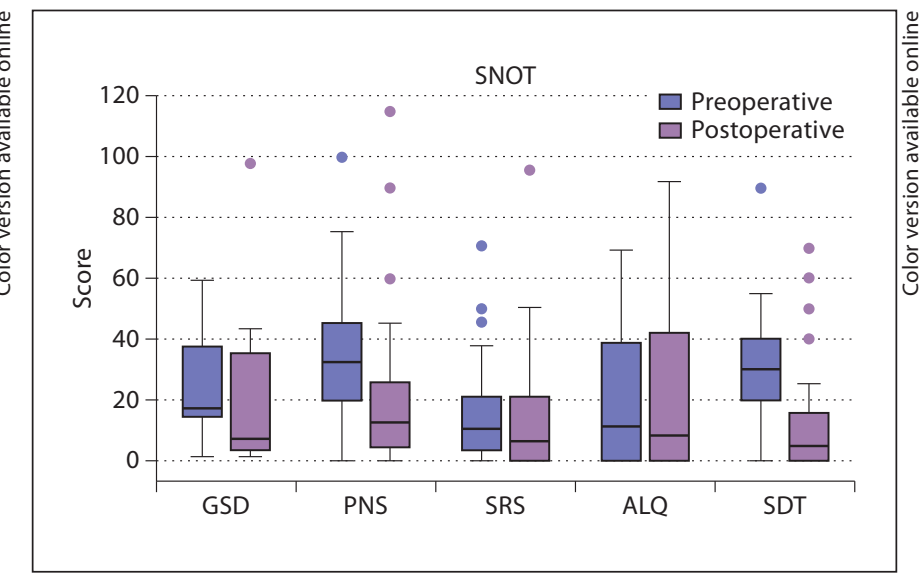

Fig. 2. The analysis of the SNOT-20 GAV SDT showed in total a significant reduction of the total score (GSD) as well as a significant reduction in both the PNS and the SDT.

Thus, with both a general assessment (GBI) and an assessment specially developed for sinonasal conditions and nasoseptal defects (SNOT-20 GAV SDT), a significant improvement was shown not only in perforationspecific and primary nasal symptoms but also in general health following a nasoseptal defect closure.

\section{Discussion}

The three-layer nasoseptal defect is a rare condition with a prevalence of $0.9 \%[1,2]$. If symptoms such as whistling noise in inspiration, multiple endonasal incrustations with impaired nasal breathing, pain or recurrent epistaxis persist despite special nasal care, a surgical septum perforation closure, if technically possible, is desirable. The superior and inferior bipedicle bridge flap technique developed by Schulz-Coulon is currently the surgical gold standard. For the success of such a gold standard procedure not only technical success but also positive changes in quality of life should be taken into consideration $[2,9,11,12]$.

The current study aims to capture the subjective results following a surgical nasoseptal defect closure. For this purpose, there are numerous validated assessments for general quality of life as well as for sinonasal conditions currently available $[19,21,23,28]$. The GBI and the SNOT-20 GAV SDT, with septal defect-specific items according to Neumann, were used in the study. This allows patient-related changes in quality of life after surgical closure of nasoseptal defects to be represented [12, 21, 28]. 
In terms of Cella's concept of patients' individual health-related quality of life, the two questionnaires combined cover all four dimensions (psychosomatic, functional, social and psychological-emotional) [29].

The psychosomatic dimension (pain, nasal breathing impairment, nosebleeds) is well represented by Neumann's expanded version of the SNOT 20 GAV SDT. The functional dimension (daily life activities) and social dimension (family relationships and friendships) are included in the 18 questions in the GBI. The psychologicalemotional dimension for capturing health-related quality of life is reflected both in the GBI and the SNOT 20 GAV SDT. Questions regarding fear, depression and sadness represent this dimension.

Thus both the GBI and the SNOT 20 GAV SDT fulfill the assessment requirement of reflecting at least three of the four dimensions $[18,28,29]$.

The evaluation of the GBI yielded a significant improvement for the total score and for the subscore 'general health' showing a positive change in the quality of life of patients following a surgical septum closure. The 18 questions on the GBI are general in terms of health problems and ENT procedures [28]. To track specific changes in otorhinolaryngological symptoms through surgical interventions on the nasal septum or nasal cavities, the SNOT 20 GAV SDT with septum defect-specific items by Neumann was added and evaluated. The patients questioned answered 25 standardized questions ranking their pre- and postoperative conditions on a scale of $0-5[12,28]$. The significant reduction in the total score, the subscore 'primary nasal symptoms' and 'septum-specific symptoms' not only shows that the rhino- genic condition generally improved, but also that defectspecific symptoms were significantly reduced.

The study thus shows that successful surgical septum perforation closure as the gold standard in septum perforation treatment leads not only to an improvement in perforation-specific and primary nasal symptoms but also to an improvement in general health and therefore to a subjective improvement in quality of life.

\section{Conclusion}

Septum perforation is a rare condition, with a prevalence of $0.9 \%$. The therapy of choice, recommended as the gold standard, is currently surgical closure using the superior and inferior bipedicle bridge flap technique according to Schulz-Coulon.

The success of a medical or surgical intervention is not only determined by the technical success of the procedure, but also by positive changes in patients' quality of life.

According to our results, successful surgical septum perforation closure leads to a significant improvement not only in perforation-specific and primary nasal symptoms, but also to an improvement in general health and therefore to a subjectively improved quality of life.

\section{Disclosure Statement}

No conflict of interest and no financial relationships with relevant commercial interests for all authors. The study was financed by the HELIOS Research support ID 011398.

\section{References}

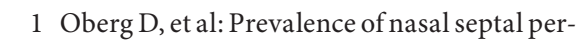
foration: the Skovde population-based study. Rhinology 2003;41:72-75.

-2 Stange T, Schultz-Coulon HJ: Closure of nasoseptal defects in Germany: the current state of the art. Laryngorhinootologie 2010; 89:157-161.

3 Dinis PB, Haider H: Septoplasty: long-term evaluation of results. Am J Otolaryngol 2002;23:85-90.

-4 Dommerby H, Rasmussen OR, Rosborg J: Long-term results of septoplastic operations. ORL J Otorhinolaryngol Relat Spec 1985;47: 151-157.

5 Konstantinidis I, et al: Long term results following nasal septal surgery. Focus on patients' satisfaction. Auris Nasus Larynx 2005;32:369-374.
6 Muhammad I, Nabil-Ur R: Complications of the surgery for deviated nasal septum. J Coll Physicians Surg Pak 2003;13:565-568.

-7 Younger R, Blokmanis A: Nasal septal perforations. J Otolaryngol 1985;14:125-131.

8 Goh AY, Hussain SS: Different surgical treatments for nasal septal perforation and their outcomes. J Laryngol Otol 2007;121:419426.

9 Kridel RW: Considerations in the etiology, treatment, and repair of septal perforations. Facial Plast Surg Clin North Am 2004;12: 435-450, vi.

10 Schultz-Coulon HJ: The bridge flap concept in closure of large defects of the nasal septum. HNO 1989;37:123-127.
11 Schultz-Coulon HJ: Three-layer repair of nasoseptal defects. Otolaryngol Head Neck Surg 2005;132:213-218.

12 Neumann A, et al: Inoperable nasoseptal defects: closure with custom-made silastic prostheses. HNO 2010;58:364-370.

13 Grutzenmacher S, et al: First findings about the nasal airflow in noses with septal perforation. Laryngorhinootologie 2002;81:276279.

14 Imperatori CJ: Diseases of the Nose and Throat. New York, Harper \& Row, 1935.

15 Kastenbauer ER, Masing H: Chirurgie der inneren Nase, Versorgung von Nasenverletzungen, vol 1. Kopf- und Halschirurgie, 1985. 
16 Baumann I: Research on quality of life in ear, nose, and throat medicine. HNO 2009;57: 855-856.

17 Carr AJ, Gibson B, Robinson PG: Measuring quality of life: Is quality of life determined by expectations or experience? BMJ 2001;322: 1240-1243.

18 Higginson IJ, Carr AJ: Measuring quality of life: using quality of life measures in the clinical setting. BMJ 2001;322:1297-1300.

19 Robinson K, Gatehouse S, Browning GG: Measuring patient benefit from otorhinolaryngological surgery and therapy. Ann Otol Rhinol Laryngol 1996;105:415-422.

$\checkmark 20$ Baumann I: Quality of life before and after septoplasty and rhinoplasty. Laryngorhinootologie 2010;89(suppl 1):S35-S45.
21 Baumann I: Validated instruments to measure quality of life in patients with chronic rhinosinusitis. HNO 2009;57:873-881.

22 Baumann I, et al: Patients with chronic rhinosinusitis: disease-specific and general health-related quality of life. HNO 2006;54: 544-549.

23 Fahmy FF, McCombe A, McKiernan DC: Sino nasal assessment questionnaire, a patient focused, rhinosinusitis specific outcome measure. Rhinology 2002;40:195-197.

24 Neumann A, et al: Patients' satisfaction after nasal septal and turbinate surgery. Results of a questionnaire. Laryngorhinootologie 2007;86:706-713.

5 Piccirillo JF, Merritt MG Jr, Richards ML: Psychometric and clinimetric validity of the 20-Item Sino-Nasal Outcome Test (SNOT20). Otolaryngol Head Neck Surg 2002;126: 41-47.
26 Bhattacharyya N, Kepnes LJ: Economic benefit of tonsillectomy in adults with chronic tonsillitis. Ann Otol Rhinol Laryngol 2002; 111:983-988.

27 Fahy C, Nikolopoulos TP, O’Donoghue GM: Acoustic neuroma surgery and tinnitus. Eur Arch Otorhinolaryngol 2002;259:299-301.

28 Baumann I, Plinkert PK, De Maddalena H Development of a grading scale for the SinoNasal Outcome Test-20 German Adapted Version (SNOT-20 GAV). HNO 2008;56: 784-788.

29 Cella D: Instruments and Assessment Methods in Psycho-Oncology Quality of Life. Textbook of Psycho-Oncology. London, Oxford University Press, 1997. 